\title{
Some combinatorial identities involving noncommuting variables
}

\author{
Michael Schlossen $\|^{\dagger}$ and Meesue Yod" \\ Fakultät für Mathematik, Universität Wien, Oskar-Morgenstern-Platz 1, A-1090 Vienna, Austria
}

\begin{abstract}
We derive combinatorial identities for variables satisfying specific sets of commutation relations. The identities thus obtained extend corresponding ones for $q$-commuting variables $x$ and $y$ satisfying $y x=q x y$. In particular, we obtain weight-dependent binomial theorems, functional equations for generalized exponential functions, we propose a derivative of noncommuting variables, and finally utilize one of the considered weight functions to extend rook theory. This leads us to an extension of the $q$-Stirling numbers of the second kind, and of the $q$-Lah numbers.

Résumé. Nous obtenons des identités combinatoires pour des variables satisfaisant des ensembles spécifiques de relations de commutation. Ces identités ainsi obtenues généralisent leurs analogues pour des variables $q$-commutantes $x$ et $y$ satisfaisant $y x=q x y$. En particulier, nous obtenons des théorèmes binomiaux dépendant du poids, des équations fonctionnelles pour les fonctions exponentielles généralisées, nous proposons une dérivée des variables non-commutatives, et finalement nous utilisons l'une des fonctions de poids considérées pour étendre la théorie des tours. Nous en déduisons une généralisation des $q$-nombres de Stirling de seconde espèce et des $q$-nombres de Lah.
\end{abstract}

Keywords: noncommuting variables, weight dependent binomial theorem, combinatorial identities

\section{Introduction}

A fundamental question of algebraic combinatorics concerns the study of connections between algebraic relations and combinatorics. For instance, the well-studied $q$-commutation relations $y x=q x y$ can be interpreted in terms of weighted lattice paths. The algebraic expression " $x y$ " would refer to a path going one step east, then one step north, while " $y x$ " would refer to a path with first step north and second step east. Keeping track of the area "covered" by the path (i.e., the number of square units below the path) and assigning the weight of a path $P$ to be $q^{a}$ where $a$ is the area of $P$, we see that the weight of the path $y x$ is $q$ whereas the weight of $x y$ is 1 , or, with other words, the path $y x$ has an additional weight $q$ compared to the path $x y$. The commutation relation $y x=q x y$ describes exactly the change of the weights when the two steps are interchanged.

Our purpose here is to carry out a similar analysis with even more general weights (which depend on the position of the steps of the path). In particular, we establish noncommutative extensions of the binomial

\footnotetext{
$\dagger^{\dagger}$ Email: michael.schlosser@univie.ac. at Partially supported by FWF Austrian Science Fund grant SFB F50 (Special Research Program "Algorithmic and Enumerative Combinatorics".

‡Email: meesue. yoo@univie. ac at Supported by FWF Austrian Science Fund grant SFB F50. 
theorem, functional equations for generalized exponentials, propose a weighted derivative, and employ one of the weight functions in rook theory by which we are led to an extension of the $q$-Stirling numbers of the second kind, and of the $q$-Lah numbers.

Overall, we expect that our findings will not only have applications to algebraic combinatorics but also to noncommutative analysis, algebraic geometry, and quantum groups.

\section{Noncommutative weight-dependent binomial theorem}

Let $\mathbb{N}$ and $\mathbb{N}_{0}$ denote the sets of positive and nonnegative integers, respectively.

Definition 2.1 For a doubly-indexed sequence of indeterminates $(w(s, t))_{s, t \in \mathbb{N}}$, let $\mathbb{C}_{w}[x, y]$ be the associative unital algebra over $\mathbb{C}$ generated by $x$ and $y$, satisfying the following three relations :

$$
\begin{aligned}
y x & =w(1,1) x y, \\
x w(s, t) & =w(s+1, t) x, \\
y w(s, t) & =w(s, t+1) y,
\end{aligned}
$$

for all $(s, t) \in \mathbb{N}^{2}$.

For $s \in \mathbb{N}$ and $t \in \mathbb{N}_{0}$, we define

$$
W(s, t):=\prod_{j=1}^{t} w(s, j),
$$

the empty product being defined to be 1 . Note that for $s, t \in \mathbb{N}$, we have $w(s, t)=W(s, t) / W(s, t-1)$. We refer to the $w(s, t)$ as small weights, whereas to the $W(s, t)$ as big weights (or column weights).

Let the weight-dependent binomial coefficients be defined by

$$
{ }_{w}\left[\begin{array}{l}
0 \\
0
\end{array}\right]=1, \quad\left[\begin{array}{l}
n \\
k
\end{array}\right]=0 \quad \text { for } n \in \mathbb{N}_{0} \text {, and } k \in-\mathbb{N} \text { or } k>n,
$$

and

$$
\left[\begin{array}{c}
n+1 \\
k
\end{array}\right]={ }_{w}\left[\begin{array}{l}
n \\
k
\end{array}\right]+{ }_{w}\left[\begin{array}{c}
n \\
k-1
\end{array}\right] W(k, n+1-k) \quad \text { for } n, k \in \mathbb{N}_{0} .
$$

These weight-dependent binomial coefficients have a combinatorial interpretation in terms of weighted lattice paths, see [5]. A lattice path is a sequence of north $(0,1)$ and east $(1,0)$ steps in the first quadrant of the $x y$-plane, starting at the origin $(0,0)$ and ending at say $(n, m)$. We give weights to such paths by assigning the big weight $W(s, t)$ to each east step $(s-1, t) \rightarrow(s, t)$ and 1 to each north step. Then define the weight of a path $P, w(P)$, to be the product of the weight of all its steps.

Given two points $A, B \in \mathbb{N}_{0}^{2}$, let $\mathcal{P}(A \rightarrow B)$ be the set of all lattice paths from $A$ to $B$, and define

$$
w(\mathcal{P}(A \rightarrow B)):=\sum_{P \in \mathcal{P}(A \rightarrow B)} w(P) .
$$


Then we have

$$
w(\mathcal{P}((0,0) \rightarrow(k, n-k)))={ }_{w}\left[\begin{array}{l}
n \\
k
\end{array}\right]
$$

as both sides of the equation satisfy the same recursion and initial condition as in (3).

Interpreting the $x$-variable as an east step and the $y$-variable as a north step, we get the following weight dependent binomial theorem.

Theorem 2.2 ([6]) Let $n \in \mathbb{N}_{0}$. Then, as an identity in $\mathbb{C}_{w}[x, y]$,

$$
(x+y)^{n}=\sum_{k=0}^{n}\left[\begin{array}{l}
n \\
k
\end{array}\right] x^{k} y^{n-k} .
$$

\section{$3 a, b$-weights}

Theorem 2.2 holds with the elliptic weights

$$
w_{a, b ; q, p}(s, t)=\frac{\theta\left(a q^{s+2 t}, b q^{2 s+t-2}, a q^{t-s-1} / b ; p\right)}{\theta\left(a q^{s+2 t-2}, b q^{2 s+t}, a q^{t-s+1} / b ; p\right)} q
$$

where $a$ and $b$ are two independent variables, while $q$ and $p$ are complex numbers with $|p|<1$, usually referred to as the base and nome, respectively, and

$$
W_{a, b ; q, p}(s, t)=\frac{\theta\left(a q^{s+2 t}, b q^{2 s}, b q^{2 s-1}, a q^{1-s} / b, a q^{-s} / b ; p\right)}{\theta\left(a q^{s}, b q^{2 s+t}, b q^{2 s+t-1}, a q^{t-s+1} / b, a q^{t-s} / b ; p\right)} q^{t},
$$

where $\theta(x ; p)$ is the modified Jacobi theta function with nome $p$, defined by

$$
\theta(x ; p)=\prod_{j \geq 0}\left(\left(1-p^{j} x\right)\left(1-p^{j+1} / x\right)\right), \quad|p|<1 .
$$

For these weights, we have the elliptic binomial coefficient

$$
\left[\begin{array}{l}
n \\
k
\end{array}\right]_{a, b ; q, p}:=\frac{\left(q^{1+k}, a q^{1+k}, b q^{1+k}, a q^{1-k} / b ; q, p\right)_{n-k}}{\left(q, a q, b q^{1+2 k}, a q / b ; q, p\right)_{n-k}} .
$$

Here, we have employed the notation for the theta shifted factorial (or $q, p$-shifted factorial), defined by

$$
(a ; q, p)_{n}= \begin{cases}\prod_{k=0}^{n-1} \theta\left(a q^{k} ; p\right), & n=1,2, \ldots, \\ 1, & n=0, \\ 1 / \prod_{k=0}^{-n-1} \theta\left(a q^{n+k} ; p\right), & n=-1,-2, \ldots\end{cases}
$$

together with

$$
\left(a_{1}, a_{2}, \ldots, a_{m} ; q, p\right)_{n}=\prod_{k=1}^{m}\left(a_{k} ; q, p\right)_{n},
$$

for compact notation. For $p=0$ we have $\theta(x ; 0)=1-x$ and, hence, $(a ; q, 0)_{n}=(a ; q)_{n}$ is a $q$-shifted factorial in base $q$. 
In fact, the elliptic binomial coefficients in (8) generalize the familiar $q$-binomial coefficients, which are obtained by letting $p \rightarrow 0, a \rightarrow 0$, then $b \rightarrow 0$, in this order.

Basic hypergeometric series (and $q$-series) are covered with great detail in Gasper and Rahman's textbook [2]; elliptic hypergeometric series are studied there in Chapter 11.

By letting $p \rightarrow 0$, the elliptic weights $w_{a, b ; q, p}(s, t)$ specialize to

$$
w(s, t)=w_{a, b ; q}(s, t)=\frac{\left(1-a q^{s+2 t}\right)\left(1-b q^{2 s+t-2}\right)\left(1-a q^{t-s-1} / b\right)}{\left(1-a q^{s+2 t-2}\right)\left(1-b q^{2 s+t}\right)\left(1-a q^{t-s-1} / b\right)} q,
$$

the associated big weights being

$$
W(s, t)=W_{a, b ; q}(s, t)=\frac{\left(1-a q^{s+2 t}\right)\left(1-b q^{2 s}\right)\left(1-b q^{2 s-1}\right)\left(1-a q^{1-s} / b\right)\left(1-a q^{-s} / b\right)}{\left(1-a q^{s}\right)\left(1-b q^{2 s+t}\right)\left(1-b q^{2 s+t-1}\right)\left(1-a q^{t-s+1} / b\right)\left(1-a q^{t-s} / b\right)} q^{t}
$$

The corresponding binomial coefficients are

$$
\left[\begin{array}{l}
n \\
k
\end{array}\right]=\left[\begin{array}{l}
n \\
k
\end{array}\right]_{a, b ; q}=\frac{\left(q^{1+k}, a q^{1+k}, b q^{1+k}, a q^{1-k} / b ; q\right)_{n-k}}{\left(q, a q, b q^{1+2 k}, a q / b ; q\right)_{n-k}}
$$

Then in the unital algebra $\mathbb{C}_{a, b ; q}[x, y]$ over $\mathbb{C}$ defined by the following commutation relations

$$
\begin{aligned}
& y x=\frac{\left(1-a q^{3}\right)(1-b q)(1-a / b q)}{(1-a q)\left(1-b q^{3}\right)(1-a q / b)} q x y, \\
& x a=q a x, \quad x b=q^{2} b x \\
& y a=q^{2} a y, \quad y b=q b y
\end{aligned}
$$

the following binomial theorem holds

$$
(x+y)^{n}=\sum_{k=0}^{n}\left[\begin{array}{l}
n \\
k
\end{array}\right]_{a, b ; q} x^{k} y^{n-k} .
$$

On the other hand, we have the following new identity which can be proved by induction on $n$.

Proposition 3.1 For any constant $c$ independent from $a, b$, we have

$$
\begin{aligned}
& \prod_{\overleftarrow{k=0}}^{n-1}\left(1-W_{a, b ; q}(1, k) c x\right) \\
& \quad=\sum_{k=0}^{n}(-c)^{k}\left[\begin{array}{l}
n \\
k
\end{array}\right]_{a, b ; q} \frac{\left(a q^{n} ; q\right)_{k}}{\left(a q^{n-k+1} ; q\right)_{k}} \frac{(b q ; q)_{k}}{\left(b q^{k} ; q\right)_{k}} \frac{\left(a / b ; q^{-1}\right)_{k}(a q / b ; q)_{n-k}}{\left(a q^{n-1} / b ; q^{-1}\right)_{k}\left(a q^{1-k} / b ; q\right)_{n-k}} x^{k}
\end{aligned}
$$

where the product (containing the left arrow $\leftarrow$ ) of noncommuting factors is carried out from right to left. 


\subsection{The $a \rightarrow 0$ case}

\subsubsection{The $b, q$-binomial theorem}

If one lets $a \rightarrow 0$ in $9 \mathrm{a}$, then the corresponding weights are

$$
\begin{aligned}
w_{0, b ; q}(s, t) & =\frac{\left(1-b q^{2 s+t-2}\right)}{\left(1-b q^{2 s+t}\right)} q, \\
W_{0, b ; q}(s, t) & =\frac{\left(1-b q^{2 s}\right)\left(1-b q^{2 s-1}\right)}{\left(1-b q^{2 s+t}\right)\left(1-b q^{2 s+t-1}\right)} q^{t} .
\end{aligned}
$$

In the unital algebra $\mathbb{C}_{0, b ; q}[x, y]$ over $\mathbb{C}$ defined by the following three relations

$$
\begin{aligned}
y x & =\frac{(1-b q)}{\left(1-b q^{3}\right)} q x y, \\
x b & =q^{2} b x, \\
y b & =q b y
\end{aligned}
$$

the following binomial theorem holds :

$$
(x+y)^{n}=\sum_{k=0}^{n}\left[\begin{array}{l}
n \\
k
\end{array}\right]_{0, b ; q} x^{k} y^{n-k}
$$

where

$$
\left[\begin{array}{l}
n \\
k
\end{array}\right]_{0, b ; q}=\frac{\left(q^{1+k}, b q^{1+k} ; q\right)_{n-k}}{\left(q, b q^{1+2 k} ; q\right)_{n-k}} .
$$

In (16), by interchanging $k$ and $n-k$ and using the relation

$$
x^{l} y^{k}=\frac{\left(b q^{1+k} ; q\right)_{2 l}}{(b q ; q)_{2 l}} q^{-k l} y^{k} x^{l}
$$

we get

$$
\begin{aligned}
(x+y)^{n} & =\sum_{k=0}^{n} \frac{\left(q^{1+n-k}, b q^{1+n-k} ; q\right)_{k}}{\left(q, b q^{1+2 n-2 k} ; q\right)_{k}} x^{n-k} y^{k} \\
& =\sum_{k=0}^{n} \frac{\left(q^{1+n-k}, b q^{1+n-k} ; q\right)_{k}}{\left(q, b q^{1+2 n-2 k} ; q\right)_{k}} \times \frac{\left(b q^{1+k} ; q\right)_{2 n-2 k}}{(b q ; q)_{2 n-2 k}} q^{-k(n-k)} y^{k} x^{n-k} \\
& =\sum_{k=0}^{n} \frac{(q ; q)_{n}}{(q ; q)_{k}(q ; q)_{n-k}} \frac{(b q ; q)_{n}}{(b q ; q)_{k}(b q ; q)_{n-k}} q^{k(k-n)} y^{k} x^{n-k}
\end{aligned}
$$

Also note that we can derive the following identity

$$
\prod_{k=0}^{n-1}\left(b y+\frac{\left(b q^{2} ; q^{-1}\right)_{k}}{\left(b ; q^{-1}\right)_{k}} x\right)=\sum_{k=0}^{n}\left[\begin{array}{l}
n \\
k
\end{array}\right]_{0, b ; q} \frac{\left(b q^{k+2} ; q\right)_{n-1}}{\left(b q^{2} ; q\right)_{n-1}} q^{k(k-n)} x^{k}(b y)^{n-k}
$$




\subsubsection{Identities for $b, q$-exponentials}

Let us define the $b, q$-exponential by

$$
e_{b, q}(z):=\sum_{n=0}^{\infty} \frac{1}{(q ; q)_{n}(b q ; q)_{n}} z^{n} .
$$

Note that when $b=0$, we get the original $q$-exponential

$$
e_{q}(z)=e_{0, q}(z)=\sum_{n=0}^{\infty} \frac{z^{n}}{(q ; q)_{n}}=\frac{1}{(z ; q)_{\infty}} .
$$

The following Proposition generalizes the well-known identity for $q$-exponentials $e_{q}(x+y)=e_{q}(x) e_{q}(y)$ (a.k.a. Cauchy identity) which was first observed by Schützenberger [7].

Proposition 3.2 In the algebra $\mathbb{C}_{0, b ; q}[x, y]$, we have

$$
e_{b, q}(x+y)=e_{b, q}(x) e_{b, q}(y) .
$$

Proof: We apply the $b, q$-binomial theorem to expand $(x+y)^{n}$ in $e_{b, q}(x+y)$. Then the left-hand side of 19. can be written as

$$
\begin{aligned}
e_{b, q}(x+y) & =\sum_{n=0}^{\infty} \frac{1}{(q ; q)_{n}(b q ; q)_{n}}(x+y)^{n} \\
& =\sum_{n=0}^{\infty} \frac{1}{(q ; q)_{n}(b q ; q)_{n}} \sum_{k=0}^{n}\left[\begin{array}{l}
n \\
k
\end{array}\right]_{0, b ; q} x^{k} y^{n-k} \\
& =\sum_{n=0}^{\infty} \frac{1}{(q ; q)_{n}(b q ; q)_{n}} \sum_{k=0}^{n} \frac{(q ; q)_{n}}{(q ; q)_{k}(q ; q)_{n-k}} \frac{(b q ; q)_{n}(b q ; q)_{2 k}}{(b q ; q)_{k}(b q ; q)_{n+k}} x^{k} y^{n-k} \\
& =\sum_{n=0}^{\infty} \sum_{k=0}^{n} \frac{1}{(q ; q)_{k}(q ; q)_{n-k}(b q ; q)_{k}\left(b q^{1+2 k} ; q\right)_{n-k}} x^{k} y^{n-k} \\
& =\sum_{n=0}^{\infty} \sum_{k=0}^{n} \frac{1}{(q, b q ; q)_{k}} x^{k} \frac{1}{(q, b q ; q)_{n-k}} y^{n-k} \\
& =e_{b, q}(x) e_{b, q}(y) .
\end{aligned}
$$

Note that we used the relation $15 \mathrm{~b}$ to exchange $x$ 's and $b$ 's.

Remark 3.3 Let us set

$$
F_{b, q}(x)=\sum_{n \geq 0} \frac{1}{(q, b q ; q)_{n}} x^{n} .
$$

Then we can verify that $F_{b, q}(x)$ satisfies the following two relations

$$
F_{b, q}(x)-F_{b, q}(q x)=\frac{1}{(1-b q)} x F_{b / q, q}(x),
$$




$$
F_{b, q}(x)-b F_{b, q}(q x)=(1-b) F_{b / q, q}(x) .
$$

Combining 20a and 20b to eliminate $F_{b / q, q}(x)$ gives

$$
\left(1-\frac{1}{(1-b q)\left(1-b q^{2}\right)} x\right) F_{b, q}(x)=\left(1-\frac{b q^{2}}{(1-b q)\left(1-b q^{2}\right)} x\right) F_{b, q}(q x),
$$

or

$$
F_{b, q}(x)=\left(1-\frac{1}{(1-b q)\left(1-b q^{2}\right)} x\right)^{-1}\left(1-\frac{b q^{2}}{(1-b q)\left(1-b q^{2}\right)} x\right) F_{b, q}(q x) .
$$

By iterating 21], we get

$$
F_{b, q}(x)=\prod_{\overrightarrow{k=0}}^{\infty}\left[\left(1-\frac{1}{(1-b q)\left(1-b q^{2}\right)} x q^{k}\right)^{-1}\left(1-\frac{b q^{2}}{(1-b q)\left(1-b q^{2}\right)} x q^{k}\right)\right],
$$

where the product (containing the right arrow $\rightarrow$ ) of noncommuting factors is carried out from left to right as $k$ increases. This gives a product form for the $b, q$-exponential $e_{b, q}(x)$.

If we put $y \frac{1}{1-b}$ for $z$ in the original $q$-exponential, we get

$$
e_{q}\left(y \frac{1}{1-b}\right)=\sum_{n=0}^{\infty} \frac{1}{(q ; q)_{n}}\left(y \frac{1}{1-b}\right)^{n}=\sum_{n=0}^{\infty} \frac{1}{(q ; q)_{n}(b q ; q)_{n}} y^{n}=e_{b, q}(y) .
$$

Hence, by the property of the original $q$-exponential, we have

$$
e_{b, q}(y)=\frac{1}{\left(y(1-b)^{-1} ; q\right)_{\infty}}
$$

when the multiplication on the right-hand side in the infinite product is done from left to right. Let $u=-x y^{-1}(1-b q)$ and $v=y(1-b)^{-1}$. Then these two new variables satisfy

$$
v u=q u v .
$$

So by the properties of the $q$-exponential (cf. [4]),

$$
\begin{aligned}
& e_{q}(u) e_{q}(v)=e_{q}(u+v), \\
& e_{q}(v) e_{q}(u)=e_{q}(u) e_{q}(-u v) e_{q}(v) .
\end{aligned}
$$

Note that $e_{q}(v)=e_{b, q}(y)$ and $-u v=x$. So we get

$$
e_{b, q}(y) e_{q}\left(-x y^{-1}(1-b q)\right)=e_{q}\left(-x y^{-1}(1-b q)\right) e_{q}(x) e_{b, q}(y) .
$$

Now

$$
e_{q}\left(-x y^{-1}(1-b q)\right)=\sum_{n=0}^{\infty} \frac{1}{(q ; q)_{n}}\left(-x y^{-1}(1-b q)\right)^{n}
$$




$$
=\sum_{n=0}^{\infty} \frac{\left(b q^{2} ; q\right)_{n}}{(q ; q)_{n}}\left(-x y^{-1}\right)^{n} .
$$

So, if we define

$$
E_{b, q}(z)=\sum_{k=0}^{\infty} \frac{\left(b q^{2} ; q\right)_{k}}{(q ; q)_{k}}(-z)^{k}
$$

then 24 can be rewritten as

$$
e_{b, q}(y) E_{b, q}\left(x y^{-1}\right)=E_{b, q}\left(x y^{-1}\right) e_{q}(x) e_{b, q}(y)
$$

\subsection{The $b \rightarrow 0$ case}

\subsubsection{The $a, q$-binomial theorem}

If one lets $b \rightarrow 0$ in $[9]$, then the corresponding weights are

$$
\begin{aligned}
w_{a, 0 ; q}(s, t) & =\frac{\left(1-a q^{s+2 t}\right)}{\left(1-a q^{s+2 t-2}\right)} q^{-1}, \\
W_{a, 0 ; q}(s, t) & =\frac{\left(1-a q^{s+2 t}\right)}{\left(1-a q^{s}\right)} q^{-t} .
\end{aligned}
$$

In the unital algebra $\mathbb{C}_{a, 0 ; q}[x, y]$ over $\mathbb{C}$ defined by the following three relations

$$
\begin{aligned}
& y x=\frac{\left(1-a q^{3}\right)}{(1-a q)} q^{-1} x y, \\
& x a=q a x, \\
& y a=q^{2} a y,
\end{aligned}
$$

the following binomial theorem holds :

$$
(x+y)^{n}=\sum_{k=0}^{n}\left[\begin{array}{l}
n \\
k
\end{array}\right]_{a, 0 ; q} x^{k} y^{n-k}
$$

where

$$
\left[\begin{array}{l}
n \\
k
\end{array}\right]_{a, 0 ; q}=\frac{\left(q^{1+k}, a q^{1+k} ; q\right)_{n-k}}{(q, a q ; q)_{n-k}} q^{k(k-n)} .
$$

\subsubsection{An a, q-derivative operator}

Definition 3.4 Define operators acting on $x$-variables only in $\mathbb{C}_{a, 0 ; q}[x]$

$$
\begin{aligned}
& \mathcal{D}_{a ; q}\left(x^{n}\right)=\frac{\left(1-q^{n}\right)\left(1-a q^{n}\right)}{(1-q)(1-a q)} q^{1-n} x^{n-1}, \\
& \mathcal{D}_{a ; q}\left(c(a, q) x^{n}\right)=c\left(a q^{-1}, q\right) \mathcal{D}_{a ; q}\left(x^{n}\right),
\end{aligned}
$$


where $c(a, q)$ is any function depending on $a$ and $q$, and

$$
\eta_{a ; q}(f(x))=y x^{-1} f(x) x y^{-1}
$$

so that

$$
\begin{aligned}
& \eta_{a ; q} x^{n}=\frac{\left(1-a q^{n+1}\right)\left(1-a q^{n+2}\right)}{(1-a q)\left(1-a q^{2}\right)} q^{-n} x^{n}, \\
& \eta_{a ; q}(c(a, q) f(x))=c(a q, q) \eta_{a ; q}(f(x)) .
\end{aligned}
$$

These operators satisfy the following relation ("Pincherle derivative"):

$$
\mathcal{D}_{a ; q} x-x \mathcal{D}_{a ; q}=\eta_{a ; q} .
$$

More generally, the following holds.

Proposition 3.5 For $k \geq 1$,

$$
\mathcal{D}_{a ; q}^{k} x-x \mathcal{D}_{a ; q}^{k}=\left[\begin{array}{c}
k \\
1
\end{array}\right]_{a q, 0 ; q} W_{a, 0 ; q}(k+1,1-k) \mathcal{D}_{a ; q}^{k-1}\left(\eta_{a ; q}\right) .
$$

Proof: We apply both sides of the operator equation to $c(a, q) x^{n}$ and verify that the results are the same.

\section{Extension of rook theory}

Here we utilize the elliptic weight defined in 6 to extend the $q$-rook polynomials. For this, we consider an $n \times n$ grid, $[n] \times[n]$, where $[n]=\{1,2, \ldots, n\}$ and label the rows from top to bottom with $1,2,3, \ldots$, and the columns from left to right with $1,2,3, \ldots$, and let $(i, j)$ denote the square in the $i$-th column from the left and $j$-th row from the top. A board is a subset of $[n] \times[n]$, and a board $B=B\left(b_{1}, \ldots, b_{n}\right)$ is called a Ferrers board if it consists of bottom-right justified cells, that is, for $b_{1} \leq b_{2} \leq \cdots \leq b_{n}$,

$$
B=B\left(b_{1}, \ldots, b_{n}\right)=\left\{(i, j) \mid 1 \leq i \leq n, n-b_{i}+1 \leq j \leq n\right\} .
$$

From now on, we only consider Ferrers boards. We say that we place $k$ nonattacking rooks in $B$ for choosing a $k$-subset of $B$ such that no two elements have a common coordinate. Let $\mathcal{N}_{k}(B)$ denote the set of all $k$-rook placements in $B$ such that no two rooks lie in the same row or column. The $k$-th rook number of $B$ is defined by $r_{k}(B)=\left|\mathcal{N}_{k}(B)\right|$. Garsia and Remmel [1] introduced a $q$-analogue of the rook numbers for Ferrers boards

$$
r_{k}(q ; B)=\sum_{P \in \mathcal{N}_{k}} q^{\operatorname{inv}(P)}
$$

where $\operatorname{inv}(P)$ counts the number of uncancelled cells in a $k$-rook placement $P$ when a rook in $P$ cancels all cells in the same row to its right, and all cells below it, and itself, and they showed that

$$
\prod_{i=1}^{n}\left[z+b_{i}-i+1\right]_{q}=\sum_{k=0}^{n} r_{n-k}(q ; B)[z]_{q} \downarrow_{k}
$$

where $[n]_{q}=\frac{1-q^{n}}{1-q}$ and $[n]_{q} \downarrow_{k}=[n]_{q}[n-1]_{q} \ldots[n-k+1]_{q}$.

Here we define an elliptic analogue of the $q$-rook number. 
Definition 4.1 Given a Ferrers board B, we define the elliptic analogue of the k-rook number by

$$
r_{k}(a, b ; q, p ; B)=\sum_{P \in \mathcal{N}_{k}(B)} w t(P),
$$

where

$$
w t(P)=\prod_{(i, j) \in U n c(P)} w_{a, b ; q, p}\left(1, i+j-1-r_{P,(i, j)}\right),
$$

$w_{a, b ; q, p}(1, k)=\frac{\theta\left(a q^{2 k+1}, b q^{k}, a q^{k-2} / b ; p\right)}{\theta\left(a q^{2 k-1}, b q^{k+2}, a q^{k} / b ; p\right)} q$, Unc $(P)$ is the set of uncancelled cells in $B$ of $P$, and $r_{P,(i, j)}$ is the number of rooks in $P$ which are in the north-west region of $(i, j)$.

Notice that in the elliptic case the weight of a rook placement $P$ is much more involved than in the much simpler $q$-case (where the quantities $r_{P,(i, j)}$ are not needed).

The $r_{k}(a, b ; q, p ; B)$ happen to satisfy an analogous identity to 32 .

Theorem 4.2 For a Ferrers board $B=B\left(b_{1}, \ldots, b_{n}\right)$, with $b_{1} \leq b_{2} \leq \cdots \leq b_{n}$, we have

$$
\begin{aligned}
& \prod_{i=1}^{n}\left[\begin{array}{c}
z+b_{i}-i+1 \\
1
\end{array}\right]_{a q^{2\left(n-b_{i}+i-1\right)}, b q^{n-b_{i}+i-1} ; q, p} \\
& =\sum_{k=0}^{n} r_{n-k}(a, b ; q, p ; B) \prod_{j=1}^{k}\left[\begin{array}{c}
z-j+1 \\
1
\end{array}\right]_{a q^{2 n+2 j-2}, b q^{n+j-1} ; q, p} .
\end{aligned}
$$

Proof: The proof is similar to the proof in the $q$-rook number case given in [1]. We extend the board by attaching an $n \times z$ rectangular shape board below the original board $B$ and denote the extended board by $B_{z}$. Say we place $n$ rooks in $B_{z}$ and compute $\sum_{P \in \mathcal{N}_{n}\left(B_{z}\right)} w t(P)$. Then the left-hand side of (33) computes it by placing rooks column-wise, and the right-hand side computes it by placing $n-k$ rooks in $B$ first, and then placing $k$ rooks in the empty columns of the $n \times z$ board below $B$. In doing so, the following lemma is crucial to get a product formula.

Lemma 4.3 Suppose that $Q \in \mathcal{N}_{t}\left(B_{z}\right)$ is a rook placement of trooks in the first $i-1$ columns of $B_{z}$. Let $D_{i}(Q)$ denote the set of all rook placements which extend $Q$ by adding a rook in column $i$. Then we have

$$
\sum_{P \in D_{i}(Q)} w t(P)=\left[\begin{array}{c}
b_{i}+z-t \\
1
\end{array}\right]_{a q^{2\left(n-b_{i}+i-1\right)}, b q^{n-b_{i}+i-1} ; q, p} w t(Q) .
$$

The above lemma can be proved easily by considering the combinatorial interpretation of the weighted binomial coefficients given in (4). The weights of the uncancelled cells add up nicely to a binomial coefficient by subtracting $r_{P,(i, j)}$ in the definition of the weight of uncancelled cells $w_{a, b ; q, p}(1, i+j-$ $\left.1-r_{P,(i, j)}\right)$. We omit the details of the proof.

As the Stirling numbers have nice rook theoretic interpretations when $B$ is a staircase board $S t_{n}=$ $B(0,1, \ldots, n-1)$, Theorem 4.2 gives an elliptic analogue of the Stirling numbers of the second kind. For $b_{i}=i-1$, 33 becomes

$$
\left(\left[\begin{array}{l}
z \\
1
\end{array}\right]_{a q^{2 n}, b q^{n} ; q, p}\right)^{n}=\sum_{k=0}^{n} r_{n-k}\left(a, b ; q, p ; S t_{n}\right) \prod_{j=1}^{k}\left[\begin{array}{c}
z-j+1 \\
1
\end{array}\right]_{a q^{2 n+2 j-2}, b q^{n+j-1} ; q, p} .
$$


If we replace $a$ by $a q^{-2 n}$ and $b$ by $b q^{-n}$ in 35 , then $r_{n-k}\left(a q^{-2 n}, b q^{-n} ; q, p ; S t_{n}\right)$ become the $e l$ liptic Stirling numbers of the second kind $\mathcal{S}_{a, b ; q, p}(n, k)$, which have recently been defined by Zsófia Kereskényiné Balogh and the first author [3]. If we denote $r_{n-k}\left(a, b ; q, p ; S t_{n}\right)$ in 35 by $S_{a, b ; q, p}(n, k)$, then by considering whether there is a rook in the last column or not, we get the following recursion

$$
S_{a, b ; q, p}(n+1, k)=W_{a q^{2 n+2}, b q^{n+1} ; q, p}(1, k-1) S_{a q^{2} ; b q ; q, p}(n, k-1)+\left[\begin{array}{l}
k \\
1
\end{array}\right]_{a q^{2 n+2}, b q^{n+1} ; q, p} S_{a q^{2}, b q ; q, p}(n, k),
$$

where $W_{a, b ; q, p}(s, t)$ is as defined in (7).

Also, by considering the board $L_{n}=B(n-1, n-1, \ldots, n-1)$, we get an elliptic analogue of the Lah numbers. With $b_{i}=n-1$ for all $i=1, \ldots, n, 33$ becomes

$$
\begin{aligned}
& {\left[\begin{array}{c}
z+n-1 \\
1
\end{array}\right]_{a q^{2}, b q ; q, p}\left[\begin{array}{c}
z+n-2 \\
1
\end{array}\right]_{a q^{4}, b q^{2} ; q, p} \cdots\left[\begin{array}{c}
z \\
1
\end{array}\right]_{a q^{2 n}, b q^{n} ; q, p}} \\
& =\sum_{k=1}^{n} r_{n-k}\left(a, q ; L_{n}\right)\left[\begin{array}{c}
z \\
1
\end{array}\right]_{a q^{2 n}, b q^{n} ; q, p}\left[\begin{array}{c}
z-1 \\
1
\end{array}\right]_{a q^{2 n+2}, b q^{n+1} ; q, p} \ldots\left[\begin{array}{c}
z-k+1 \\
1
\end{array}\right]_{a q^{2 n+2 k-2, b q^{n+k-1} ; q, p}}
\end{aligned}
$$

Let $\mathcal{L}_{n, k}(a, b ; q, p)$ denote $r_{n-k}\left(a, b ; q, p ; L_{n}\right)$. Then according to whether there is a rook in the union of the top row and the last column of the board $L_{n}$ or not, we get the following recursion for $\mathcal{L}_{n, k}(a, b ; q, p)$

$$
\mathcal{L}_{n+1, k}(a, b ; q, p)=W_{a q^{2}, b q ; q}(1, n+k-1) \mathcal{L}_{n, k-1}\left(a q^{2}, b q ; q, p\right)+\left[\begin{array}{c}
n+k \\
1
\end{array}\right]_{a q^{2}, b q ; q, p} \mathcal{L}_{n, k}\left(a q^{2}, b q ; q, p\right)
$$

If we let $p=0$ and $b \rightarrow 0$, then the $a, q$-analogues of the Lah numbers have the following closed form

$$
\mathcal{L}_{n, k}(a, q)=q^{\left(\begin{array}{c}
k \\
2
\end{array}\right)-\left(\begin{array}{c}
n \\
2
\end{array}\right)-n(k-1)}\left[\begin{array}{c}
n \\
k
\end{array}\right]_{q} \frac{[n-1]_{q} !}{[k-1]_{q} !} \frac{\left(a q^{n+k+1} ; q\right)_{n+k}}{\left(a q^{3} ; q^{2}\right)_{n}\left(a q^{2+2 n} ; q^{2}\right)_{k}}
$$

(the elliptic Lah numbers don't have closed form), which converge to the $q$-Lah numbers when $a \rightarrow \infty$. Kereskényiné Balogh and the first author [3] also have arrived at an elliptic analogue of Lah numbers which are consistent with the above $\mathcal{L}_{n, k}(a, b ; q, p)$ when $a$ is replaced by $a q^{-2 n}$ and $b$ is replaced by $b q^{-n}$. Note that we can get the $a, b$-weighted version of Stirling numbers and Lah numbers by letting $p=0$.

\section{Remarks}

1. The $a$-weight can be expressed as

$$
w_{a, 0 ; q}(s, t)=\frac{\left(1-a q^{s+2 t}\right)}{\left(1-a q^{s+2 t-2}\right)} q^{-1}=\frac{q^{-\frac{s}{2}-t} / \sqrt{a}-\sqrt{a} q^{\frac{s}{2}+t}}{q^{-\frac{s}{2}-t+1} / \sqrt{a}-\sqrt{a} q^{\frac{s}{2}+t-1}} .
$$

(The choice of the square root of $a$ does not matter, as long as it is the same everywhere.) If we let $q=e^{i x}, \sqrt{a}=e^{i(\alpha+1) x}$, then

$$
w_{a, 0 ; q}(s, t)=\frac{e^{-i\left(\alpha+\frac{s}{2}+t+1\right) x}-e^{i\left(\alpha+\frac{s}{2}+t+1\right) x}}{e^{-i\left(\alpha+\frac{s}{2}+t\right) x}-e^{i\left(\alpha+\frac{s}{2}+t\right) x}}=\frac{\sin \left(\alpha+\frac{s}{2}+t+1\right) x}{\sin \left(\alpha+\frac{s}{2}+t\right) x}
$$




$$
=\frac{U_{\alpha+\frac{s}{2}+t}(x)}{U_{\alpha+\frac{s}{2}+t-1}(x)},
$$

where $U_{n}(\cos \theta)=\frac{\sin (n+1) \theta}{\sin \theta}$ is the Chebyshev polynomial of the second kind. Hence the $a$-weight $w_{a, 0 ; q}(s, t)$ can be considered as a quotient of generalized Chebyshev polynomials. Thus the identities related to the $a$-weights can be reformulated as identities for Chebyshev polynomials of the second kind.

2. It is important to realize that every identity involving the variables $a, b, x$ and $y$ respecting the commutative relations (11) also holds when they are replaced by the variables $b, a, y$ and $x$, respectively, due to the symmetry of 11 .

In this way we can immediately deduce various additional results such as $a, q$-versions from corresponding $b, q$-versions (and vice-versa), etc. To single out a particular result, we note that the following functional equation of $a, q$-exponentials

$$
e_{a, q}(x+y)=e_{a, q}(y) e_{a, q}(x)
$$

holds where, as in (18),

$$
e_{a, q}(z):=\sum_{n=0}^{\infty} \frac{1}{(q ; q)_{n}(a q ; q)_{n}} z^{n}
$$

3. We were able to establish functional equations for $a, q$ - and for $b, q$-exponentials. As a matter of fact, we were not able to unify both results and obtain a nice functional equation for $a, b, q$-exponentials.

\section{References}

[1] A. M. Garsia and J. B. Remmel, " $Q$-counting rook configurations and a formula of Frobenius", $J$. Combin. Theory Ser. A 41 (1986), 246-275.

[2] G. Gasper and M. Rahman, Basic hypergeometric series, second edition, Encyclopedia of Mathematics and Its Applications 96, Cambridge University Press, Cambridge, 2004.

[3] Z. R. Kereskényiné Balogh and M. J. Schlosser, "Elliptic Stirling numbers of the first and second kind", in preparation.

[4] T.H. Koornwinder, "Special functions and $q$-commuting variables", in Special functions, q-series and related topics (Toronto, ON, 1995), pp. 131-166, Fields Inst. Commun. 14, Amer. Math. Soc., Providence, RI, 1997.

[5] M. J. Schlosser, "Elliptic enumeration of nonintersecting lattice paths", J. Combin. Theory Ser. A 114 (2007), 505-521.

[6] M. J. Schlosser, "A noncommutative weight-dependent generalization of the binomial theorem", preprint arXiv:1106.2112.

[7] M.P. Schützenberger, "Une interprétation de certaines solutions de l'équation fonctionnelle: $F(x+$ $y)=F(x) F(y)$ ". C. R. Acad. Sci. Paris 236, (1953), 352-353. 